Quim. Nova, Vol. 35, No. 8, 1542-1548, 2012

\title{
ISOLAMENTO E ANÁLISE QUÍMICA PARCIAL DE EXOPOLISSACARÍDEOS DA DIATOMÁCEA MARINHA CULTIVADA Coscinodiscus wailesii (Coscinodiscales, Bacillariophyta)
}

\author{
Poliana G. Marson-Ascêncio* e Sérgio Donizeti Ascêncio \\ Universidade Federal do Tocantins, Campus Universitário de Palmas, Av. NS15, B1. BALA-Medicina, 77020-210 Palmas - TO, Brasil \\ Selma Faria Zawadzki Baggio \\ Departamento de Bioquímica e Biologia Molecular, Setor de Ciências Biológicas, Universidade Federal do Paraná, Centro \\ Politécnico, CP 19046, 81531-990 Curitiba-PR, Brasil
}

Recebido em 15/11/11; aceito em 9/4/12; publicado na web em 3/7/12

\begin{abstract}
ISOLATION AND PARTIAL CHEMICAL ANALYSIS OF EXOPOLYSACCHARIDES FROM CULTIVATED MARINE DIATOM Coscinodiscus wailesii (Coscinodiscales, Bacillariophyta). The marine diatom Coscinodiscus wailesii has attracted ecological interest because their blooms affect fishing areas. The aim of this work was the isolation, extraction and partial chemical characterization of soluble exopolysaccharide and bound exopolysaccharide from $C$. wailesii. Cultures were grown in Guillards f/2 medium under controlled conditions of temperature, aeration, photoperiod and light intensity. Percentage of carbohydrate, uronic acids, sulfates groups and cellular lipids was determined. Ion exchange chromatography of exopolysaccharides produced three fractions whose partial chemical structures were disclosed using ${ }^{13} \mathrm{C}$ NMR and methylation techniques.
\end{abstract}

Keywords: Coscinodiscus wailesii; diatom; exopolysaccharides.

\section{INTRODUÇÃO}

As florações de algumas espécies de microalgas podem trazer consequências negativas ao ecossistema prejudicando áreas de pesca e cultivo comerciais, através da produção de toxinas, mucilagens e várias alterações nas cadeias tróficas aquáticas. ${ }^{1}$

A microalga marinha Coscinodiscus wailesii Gran et Angst, uma diatomácea cêntrica, quando em floração pode diminuir o índice de nutrientes e de oxigênio da água, provocando a morte ou migração de peixes. Efeitos desta espécie no ecossistema resultaram em sérios danos em culturas de "Nori" (Porphyra), macroalga marinha muito utilizada comercialmente como alimento, devido à absorção acelerada de nutrientes por esta diatomácea durante sua floração na costa japonesa. $^{2}$

A distribuição da espécie $C$. wailesii, antes restrita ao Pacífico Tropical e ao Atlântico Ocidental, ampliou-se até a Europa, Estados Unidos, Japão e Austrália, tornando-se, em algumas áreas, importante componente da comunidade fitoplanctônica. ${ }^{3}$ No Brasil, na Baía de Paranaguá - PR, a espécie C. wailesii tem sido encontrada desde 1991 em baixas concentrações em todos os meses do ano, produzindo florações esporadicamente, durante as quais se observou uma marcada diminuição da população do fitoplâncton e zooplâncton. Embora a relação entre esses eventos ainda não tenha sido explicada, a possibilidade dessa microalga causar efeitos nocivos motiva a realização de estudos sobre essa espécie ecologicamente importante. ${ }^{4}$

Vários autores divergem quanto à composição do polissacarídeo da cápsula mucilaginosa de microalgas ser diferente ou não da composição do exopolissacarídeo solúvel no meio de cultura. Experimentos distintos com diatomáceas do gênero Chaetoceros, com as espécies Cylindrotheca closterium, Nitzschia sp. e Navicula salinarum, mostraram que os exopolissacarídeos produzidos são diferentes daqueles constituintes da cápsula mucilaginosa, descartando a possibilidade dos polissacarídeos encontrados no meio serem provenientes da morte ou degradação celular durante a fase estacionária do cultivo. ${ }^{5,6} \mathrm{Os}$ autores afirmam que essas diferenças na composição, localização e

*e-mail: polianamarson@uft.edu.br produção dos polissacarídeos sugerem que os polímeros servem para diferentes propósitos e que, provavelmente, possuem um controle metabólico diferente. ${ }^{6}$

Desta forma, foram avaliados os polissacarídeos extracelulares solúveis e os polissacarídeos da cápsula mucilaginosa de $C$. wailesii, uma vez que não há estudos sobre esses polissacarídeos em condições padronizadas de cultivo. O presente estudo visa contribuir para o melhor entendimento dos danos causados ao ecossistema marinho, uma vez que esses já foram atribuídos à grande quantidade de mucilagem produzida por essa espécie de diatomácea., ${ }^{1,7}$

\section{PARTE EXPERIMENTAL}

\section{Cultivo da microalga marinha Coscinodiscus wailesii}

A microalga foi coletada na Baía de Paranaguá e classificada por Fernandes. ${ }^{4} \mathrm{O}$ cultivo foi realizado em meio Guillard-f/ $2,{ }^{8}$ temperatura de $22 \pm 1{ }^{\circ} \mathrm{C}$, intensidade de irradiação luminosa de $45 \mathrm{~mol} \mathrm{~m}^{-2} \mathrm{~s}^{-1} \mathrm{e}$ fotoperíodo de $12 \mathrm{~h}$.

\section{Descontaminação e adaptação das células de C. wailesii em micro e macrocultivos}

As células de C. wailesii foram descontaminadas por isola-

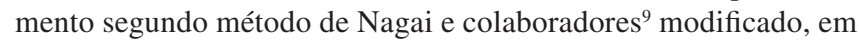
placa de 24 poços contendo meio f/2, com auxílio de lupa e pipeta de Pasteur. Uma única célula foi colocada no $1^{\circ}$ poço e sequencialmente transferida entre os poços, até o $24^{\circ}$. Após a lavagem e descontaminação, as células foram submetidas ao cultivo. Esta técnica foi utilizada uma vez que o uso de antibióticos (kanamicina, eritromicina e penicilina $\mathrm{G}$ ) impedia o desenvolvimento celular. As culturas unialgais foram confirmadas por coloração de Gram. Após o período de adaptação e multiplicação, as células foram transferidas para placas de poços maiores. O tempo necessário de adaptação em cada placa foi de aproximadamente 2 semanas. Em seguida, as células foram sequencialmente cultivadas em erlenmeyers de 250, 500 e $2000 \mathrm{~mL}$, sob aeração até se adaptarem para o cultivo 
em balões de $12 \mathrm{~L}$, contendo $7 \mathrm{~L}$ de meio de cultura, sob aeração constante, os quais foram usados para a determinação da curva de crescimento e obtenção de biomassa.

\section{Curva de crescimento}

A curva de crescimento foi determinada usando culturas em triplicata, sendo o inóculo inicial (dia zero), de aproximadamente 2 células $/ \mathrm{mL}$ de meio de cultivo, obtidas de cultura em fase logarítmica de crescimento. Para a contagem das células foram coletados $5 \mathrm{~mL}$ do meio de cultura, após agitação dos balões de cultivo. Esse procedimento foi feito em dias alternados, até o $20^{\circ}$ dia. $\mathrm{O}$ volume coletado foi fracionado em alíquotas de $0,5 \mathrm{~mL}$ em placas de 12 poços e as células visualizadas e contadas com auxílio de lupa (aumento de 300x). O número de células foi obtido pela média de 10 contagens. O cálculo do tempo de geração (TG) foi realizado segundo Shapiro ${ }^{10}$ e o cálculo para $\mu$ máx segundo EPA. ${ }^{11}$ Durante o experimento foi monitorada a produção de açúcar total e proteína conforme técnica descrita em Métodos analíticos.

\section{Extração dos polissacarídeos}

A fração polissacarídica extracelular foi obtida por filtração das culturas em funil de Büchner. O filtrado foi concentrado em evaporador rotatório sob pressão reduzida à temperatura de $50{ }^{\circ} \mathrm{C}$, dialisado (cutoff 12-14 kDa) e liofilizado dando origem à fração EXO. A massa celular retida (R I) foi submetida à extração aquosa por $1 \mathrm{~h}$, a $80^{\circ} \mathrm{C}$. Este material foi filtrado (R II), dialisado (cutoff $12-14 \mathrm{kDa}$ ), concentrado e liofilizado dando origem à fração EAQ, que contém os polissacarídeos da cápsula mucilaginosa.

\section{Extração e caracterização de lipídeos}

A massa celular ( $\mathrm{R}$ II) foi submetida à extração em $\mathrm{CHCl}_{3} /$ $\mathrm{MeOH}(2: 1)$ em sistema de refluxo a $80{ }^{\circ} \mathrm{C}$. Foram realizadas três extrações de $2 \mathrm{~h}$ cada. Os extratos foram reunidos, filtrados a quente, evaporados à temperatura ambiente e submetidos à metanólise com solução de $\mathrm{MeOH} /$ benzeno/ $\mathrm{H}_{2} \mathrm{SO}_{4}(20: 1: 1)$ por $2 \mathrm{~h}$ a $80{ }^{\circ} \mathrm{C}$. Após resfriamento, adicionou-se água destilada sob agitação constante. Os ésteres metílicos resultantes foram extraídos com hexano ( 3 ciclos), concentrados sob um fluxo de azoto para a evaporação do solvente. Os ácidos graxos derivatizados na forma de ésteres metílicos foram analisados por CG-EM, sendo a identificação dos compostos baseada nos tempos de retenção e no perfil de fragmentação de massa de padrões autênticos. Ésteres metílicos de ácido palmítico $(\geq 99 \%)$, ácido mirístico ( $\geq 99 \%$ ), ácido esteárico $(\geq 98,5 \%)$ e ácido behênico $(\geq 95,5 \%)$ foram adquiridos de Sigma-Aldrich Chemie $\mathrm{GmbH}$, Steinhein, Germany. As percentagens relativas dos ésteres metílicos presentes na amostra foram calculadas por integração de áreas no cromatograma e os resultados expressos em mol\%.

\section{Métodos analíticos}

A dosagem de proteínas foi realizada pelo método de Bradford $^{12} \mathrm{e}$ a dosagem de açúcares totais pelo método de Dubois. ${ }^{13}$ Para as curvas padrões utilizou-se albumina e glicose, respectivamente. A determinação de grupamentos sulfatos foi feita pelo método de Dodgson ${ }^{14}$ e a dosagem de ácidos urônicos pelo método do $m$-hidroxibifenil. ${ }^{15}$ Para a curva padrão utilizou-se ácido glicurônico. A desproteinização das frações polissacarídicas foi feita pela adição de ácido tricloroacético (TCA 20\% 1:1 v/v). A solução obtida foi centrifugada e o sobrenadante neutralizado com $\mathrm{NaOH} 5 \%$, dialisado contra água destilada (cutoff 12-14 kDa) e liofilizado.

\section{Redução e acetilação dos produtos de hidrólise}

A curva de hidrólise dos polissacarídeos foi monitorada pelo perfil cromatográfico obtido por CLAE nos tempos de 6, 8, 12 e $18 \mathrm{~h}$ (material suplementar). Nesses tempos, não foi observada a geração de produtos de degradação dos monossacarídeos (furfural e metilfurfural), sendo o tempo de $18 \mathrm{~h}$ o mais eficiente. Os produtos de hidrólise foram reduzidos com boro-hidreto de sódio por $12 \mathrm{~h}$ à temperatura ambiente e tratados com resina Lewatit, forma $\mathrm{H}^{+}$. Após a filtração, as soluções foram concentradas em evaporador rotativo a $50{ }^{\circ} \mathrm{C}$ até secura. $\mathrm{O}$ ácido bórico formado foi eliminado na forma de seu éster volátil por sucessivas codestilações com $\mathrm{MeOH}$. Os alditóis formados foram submetidos à acetilação com anidrido acético e piridina $(1: 1 \mathrm{v} / \mathrm{v})$ durante $24 \mathrm{~h}$ à temperatura ambiente. A reação foi interrompida pela adição de gelo pulverizado e os alditóis acetilados extraídos com $\mathrm{CHCl}_{3}$. A fase clorofórmica foi lavada com água destilada e solução de sulfato de cobre a 5\%, levada a seco e submetida à análise de CG e CG-EM.

\section{Metilação}

A metilação das frações polissacarídicas foi realizada segundo Ciucanu e Kerek, ${ }^{16}$ através da adição alternada de $\mathrm{NaOH}$ pulverizado e iodeto de metila. Foram realizados dois ciclos de metilação. As frações foram submetidas à hidrólise de Saeman, ${ }^{17}$ reduzidas, acetiladas e analisadas por CG e CG-EM na forma de acetatos de alditóis parcialmente metilados.

\section{Análises cromatográficas}

\section{Cromatografia líquida de alta eficiência (CLAE)}

Os cromatogramas foram obtidos em aparelho Shimadzu equipado com detector de índice de refração (RID 10A). Foi utilizada coluna HPX-87H 300 x 7,80 mm (AMIINEX ÍON EXCLUSION-BIO RAD) a $40{ }^{\circ} \mathrm{C}$ e fase móvel ácido sulfúrico $8 \mathrm{mM} \mathrm{L}^{-1} \mathrm{em} \mathrm{H}_{2} \mathrm{O} \mathrm{mQ}$ (saturada com gás hélio) em sistema isocrático com fluxo de $0,6 \mathrm{~mL} \mathrm{~min}^{-1}$. Os tempos de retenção dos monossacarídeos das amostras foram comparados com padrões autênticos. As percentagens relativas dos monossacarídeos das amostras foram calculadas por integração de áreas, sendo feito o ajuste de resposta ao detector para cada monossacarídeo.

\section{Cromatografia gasosa (CG)}

As análises foram realizadas em cromatógrafo Hewlett Packard, modelo 5890, acoplado a detector FID $\left(300^{\circ} \mathrm{C}\right)$. Foi utilizada coluna capilar de sílica fundida $30 \mathrm{~m}$ x 0,25 mm d.i., revestida com DB-225 da marca J.W. Foi utilizado nitrogênio como gás de arraste. A temperatura do injetor foi de $250{ }^{\circ} \mathrm{C}$ e os cromatogramas dos acetatos de alditóis foram obtidos a $220^{\circ} \mathrm{C}$.

\section{Cromatografia gasosa acoplada à espectrometria de massa (CG-EM)}

As análises foram realizadas em cromatógrafo Varian, modelo 3.300, acoplado a espectrômetro de massa da marca Finnigian Mat, modelo ITD 800, equipado com coluna capilar de sílica fundida $30 \mathrm{~m}$ x 0,25 mm d.i., modelo DB-225 da marca J.W. Foi utilizado hélio como gás de arraste. A temperatura do injetor foi de $250^{\circ} \mathrm{C}$. Os espectros de massa foram obtidos por impacto de elétrons a $70 \mathrm{meV}$ a cada $1 / 8$ de segundo, na faixa de 90 a $550 \mathrm{~m} / \mathrm{z}$

\section{Cromatografia de filtração em gel (CFG)}

As análises de homogeneidade foram realizadas em cromatógrafo de exclusão estérea de alta pressão (HPSEC) Waters, equipado com detector de índice de refração diferencial Waters modelo 2410 
e detector de espalhamento de luz em multiângulos (Malls Wyatt Technology) modelo DAWN DSP, com 18 detectores dispostos ao redor da fotocélula em diferentes ângulos. Utilizou-se o programa Astra (Wyatt Techonology) para analisar os dados obtidos. Foi efetuada a medida do espalhamento do feixe luminoso em função da concentração do polímero e em função do ângulo do feixe luminoso.

\section{Cromatografia de troca iônica em coluna de DEAE-Sephacel}

As análises foram realizadas em coluna de vidro DEAE-Sephacel na forma de sal de trietilamônio. A amostra foi eluída em gradiente descontínuo de $\mathrm{NaCl}(0,1 ; 0,2 ; 0,5 ; 1,0 ; 1,5$ e 2,0 M). As subfrações obtidas foram concentradas, dialisadas (cutoff 12-14 kDa) contra água destilada e liofilizadas.

\section{Análise de ressonância magnética nuclear}

Os espectros de ressonância magnética nuclear foram obtidos em espectrômetro da marca Bruker, série Avance, modelo DRX400. As amostras foram solubilizadas em $0,5 \mathrm{~mL}$ de $\mathrm{D}_{2} \mathrm{O}(98 \%)$, colocadas em tubos de $5 \mathrm{~mm}$ de diâmetro e analisadas à temperatura de $70{ }^{\circ} \mathrm{C}$.

Os espectros de RMN de ${ }^{13} \mathrm{C}$ (normal e DEPT135) foram obtidos na frequência base de $100,16 \mathrm{MHz}$ com janela espectral de $220 \mathrm{ppm}$ e resolução de $32 \mathrm{~K}$. Utilizou-se o intervalo de $0,6 \mathrm{~s}$ entre os pulsos para as aquisições dos sinais, sendo feitas, em média, $50 \mathrm{~K}$ aquisições. Os deslocamentos químicos foram expressos em ppm e calibrados com acetona $(30,200 \mathrm{ppm})$.

\section{RESULTADOS E DISCUSSÃO}

A análise da curva de crescimento da microalga C. wailesii em condições padronizadas de cultivo permitiu determinar o melhor período de coleta do material para estudo químico (fase estacionária, $14^{\circ}$ dia), onde é observada viabilidade celular e maior produção de carboidrato total no meio de cultura (Figura 1).

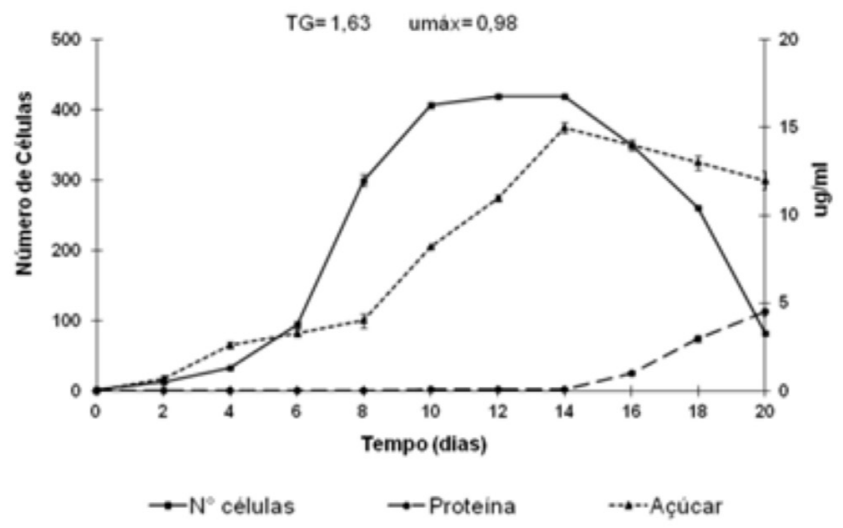

Figura 1. Curva de crescimento da diatomácea Coscinodiscus wailesii versus concentração de proteínas e carboidratos em $\mu \mathrm{g} / \mathrm{mL}$ de meio $\mathrm{f} / 2$ aerado

A quantidade de proteína solúvel no meio de cultura entre os estágios de crescimento da microalga foi insignificante, ocorrendo aumento apenas a partir do $16^{\circ}$ dia de cultivo, provavelmente por morte celular referente à fase de declínio. Esses dados descartam morte celular nas fases exponencial e estacionária, o que comprova que a dosagem de carboidrato total é decorrente apenas de exopolissacarídeos secretados pelas células.

\section{Análise dos ácidos graxos produzidos pela diatomácea $C$. wailesii}

O perfil de fragmentação dos espectros de massa de ésteres metílicos de ácidos graxos presentes no cromatograma obtido por CG-EM demonstra que a diatomácea $C$. wailesii sintetiza ácidos graxos saturados, não sendo evidenciada a presença de ácidos graxos insaturados. Os ácidos graxos encontrados na forma de ésteres metílicos foram: ácido palmítico (55 mol\%), ácido mirístico (33 mol\%), ácido esteárico (12 mol\%) e traços de ácido behênico, sendo o primeiro relato na literatura da composição de ácidos graxos para a espécie $C$. wailesii. Estes dados são extremamente importantes para os estudos de microalgas marinhas, uma vez que a biossíntese destas moléculas em diatomáceas pode estar relacionada com processos de adaptações fisiológicas, principalmente aqueles envolvendo fluidez de membrana quando as células são expostas a diferentes condições de temperatura e salinidade. ${ }^{4}$

Os resultados aqui apresentados poderão ser úteis para correlações em estudos visando explicar a ampliação da distribuição geográfica que vem sendo observada para $C$. wailesii em regiões de climas diferentes $^{3}$ e seus efeitos nocivos ao ecossistema marinho. ${ }^{4}$

\section{Análises químicas das frações polissacarídicas obtidas da diatomácea Coscinodiscus wailesii}

Os rendimentos obtidos das frações polissacarídicas brutas (não desproteinizadas) da microalga $C$. wailesii foram 31 e $10 \mathrm{mg} / \mathrm{L}$, frações EXO e EAQ, respectivamente. A baixa massa obtida, aliada à complexidade destes polímeros, retrata a dificuldade em se trabalhar com carboidratos de microalgas marinhas.

A presença de componentes como ácidos urônicos, piruvato e grupamento sulfato indica a natureza ácida dos exopolissacarídeos de microalgas, que têm sido observados em biofilmes produzidos por bactérias e diatomáceas. ${ }^{18}$ Neste estudo foi observada a presença de ácidos urônicos e altas porcentagens de grupamentos sulfatos, demonstrando a natureza ácida dos exopolissacarídeos produzidos por $C$. wailesii. A Figura 2 mostra a porcentagem de carboidrato total, ácido urônico e sulfato das frações EXO e EAQ. A presença de compostos ácidos nos exopolissacarídeos de células de $C$. wailesii também foi demonstrada por coloração de Alcian Blue ${ }^{19}$ (material suplementar).

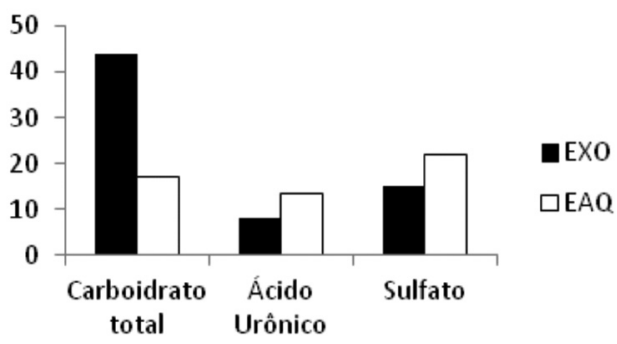

Figura 2. Porcentagem ( $\mathrm{g} \%$ ) de carboidrato total, ácido urônico e sulfato das frações EXO e EAQ obtidas de cultivo da microalga C. wailesii

Os polissacarídeos sulfatados são reportados como tendo um papel importante em oferecer flexibilidade e proteção ao dessecamento das células de microalgas, quando submetidas a condições adversas devido a variações sazonais e de marés. ${ }^{20}$

\section{Determinação da composição monossacarídica das frações EXO e EAQ}

A Tabela 1 apresenta a composição monossacarídica das frações polissacarídicas brutas obtidas da microalga $C$. wailesii cultivada.

Percival e colaboradores ${ }^{21}$ analisaram os polissacarídeos de $C$. wailesii, ainda classificada como $C$. nobilis, coletada no oeste do Canal Inglês no Reino Unido, a partir do extrato da lama coletada do 
Tabela 1. Composição monossacarídica das frações obtidas de C. wailesii (mol\%)

\begin{tabular}{cccccccccc}
\hline Fração & Glc & Man & Gal & Xyl & Fuc & Rha & Ara & GalA & GalNAc \\
\hline EXO & 20 & 18 & 29 & 1 & 16 & 7 & 3 & 4 & 2 \\
EAQ & 14 & 35 & 10 & 3 & 19 & 10 & - & 9 & - \\
\hline
\end{tabular}

- = não detectado

fundo do mar e do exopolissacarídeo produzido por essa diatomácea cultivada em meio Erd-Schreiber por 10 semanas, em condição de cultivo não padronizada. As principais diferenças encontradas no exopolissacarídeo da microalga $C$. wailesii obtido nesse trabalho e o realizado por Percival e colaboradores ${ }^{21}$ são a porcentagem de galactose, o tipo de ácido urônico e a presença ou não de açúcar aminado. A galactose é o principal monossacarídeo encontrado em nosso estudo, sendo relatado apenas traços por Percival e colaboradores, ${ }^{21} \mathrm{o}$ qual apresentou como monossacarídeo majoritário a fucose $(34 \%)$. Outra diferença é a detecção de arabinose no presente estudo. Levando em consideração o método de obtenção das amostras descrito por aqueles autores, pode-se argumentar que os polissacarídeos obtidos de amostras de lama do fundo do mar seria uma mistura de carboidratos oriundos de fontes diversas, não representando apenas os exopolissacarídeos produzidos por $C$. wailesii, o que poderia explicar as diferenças encontradas. Além disso, o uso de técnicas analíticas de alta sensibilidade e confiabilidade aqui empregadas contribui para maior riqueza de dados e segurança nos resultados apresentados.

Diferentemente de trabalhos com outras diatomáceas que apresentaram o ácido glicurônico como principal monossacarídeo ácido, a C. wailesii apresentou como único açúcar carboxilado o ácido galacturônico, detectado através de análise por CLAE. Essa diferença também é observada nos estudos de Percival e colaboradores, ${ }^{21}$ onde relataram a presença de ácido glicurônico no exopolissacarídeo de C. wailesii, sendo que, nesse caso, o açúcar ácido foi detectado por eletroforese de papel. A técnica de CLAE é uma poderosa ferramenta empregada no estudo de carboidratos, sendo considerada referência para este tipo de estudo. Sua alta sensibilidade quando comparada à eletroforese em papel sugere que possa ter havido um equívoco nos resultados apresentados por Percival e colaboradores ${ }^{21}$ em relação ao ácido glicurônico.

Um dado significativo na composição monossacarídica descrita neste trabalho é a presença de $\mathrm{N}$-acetil-galactosamina, sendo a primeira vez que se relata a presença de açúcar aminado em polissacarídeos de diatomáceas. Faz-se necessário ressaltar que açúcares aminados não são detectados por técnicas convencionais de CG, empregadas na maioria dos trabalhos publicados referentes a polissacarídeos de diatomáceas. No presente estudo, o uso de CLAE permitiu a detecção de $\mathrm{N}$-acetil-galactosamina, sendo confirmada sua presença nos polissacarídeos através da técnica de $\mathrm{RMN}$ de ${ }^{13} \mathrm{C}$. Uma revisão dos trabalhos já publicados utilizando CLAE como método para avaliar a composição monossacarídica das microalgas já estudadas poderia alterar o cenário da presença de açúcares aminados em diatomáceas.

A diferença na composição monossacarídica do exopolissacarídeo secretado por $C$. wailesii estudada neste trabalho quando comparado com o exopolissacarídeo da mesma espécie estudada por Percival e colaboradores ${ }^{21}$ pode ser explicada pelas diferenças nos cultivos, principalmente pela padronização e curva de crescimento da microalga. Como não houve padronização de cultivo nos estudos realizados por esses pesquisadores ${ }^{21}$ deve ser considerada a probabilidade da composição monossacarídica encontrada para o exopolissacarídeo ser a soma do exopolissacarídeos mais os carboidratos intracelulares e de reserva, originados de células rompidas.

A composição do exopolissacarídeo da cápsula mucilaginosa produzido pela diatomácea $C$. wailesii é quantitativa e qualitativamente diferente da encontrada para o exopolissacarídeo solúvel, como mostra a Tabela 1, portanto, para a diatomácea $C$. wailesii estes dois carboidratos são polímeros distintos.

\section{Fracionamento da fração EXO por cromatografia de troca- iônica - DEAE-Sephacel}

A Tabela 2 mostra as diferenças na composição monossacarídica das frações polissacarídicas produzidas por $C$. wailesii obtidas por cromatografia em coluna de troca-iônica DEAE-Sephacel.

Os resultados obtidos da cromatografia de exclusão estérea mostraram que estas subfrações se apresentam não homogêneas, sendo necessários passos adicionais de purificação para se propor uma estrutura com melhor definição. Porém, é possível observar a presença de polímeros distintos, sendo dados inéditos para essa espécie.

\section{Análise química parcial da fração EXO-0,1}

A atribuição de sinais no espectro de $\mathrm{RMN}$ de ${ }^{13} \mathrm{C}$ descrita aqui foi realizada considerando a composição monossacarídica dos polímeros obtidos (Tabela 2) e a intensidade dos sinais nos espectros, comparada com sinais característicos já descritos na literatura. A fração EXO-0,1 apresentou galactose como monossacarídeo principal do polímero. A análise de $\mathrm{RMN}$ de ${ }^{13} \mathrm{C}$ dessa fração mostra uma grande variedade de sinais (Figura 3). A análise da região anomérica do espectro de $\mathrm{RMN}$ de ${ }^{13} \mathrm{C}$ mostra sinais mais intensos em 103,4 e 100,1 ppm, que podem ser atribuídos ao C-1 da unidade de $\beta$-D-galactopiranose e C-1 de unidade $\alpha$-L-galactopiranose, respectivamente. ${ }^{22}$ Estas atribuições de sinais estão de acordo com os propostos por Gorin e Mazurek ${ }^{22}$ para padrões de metil- $\beta$-Dgalactopiranosídeo e metil- $\alpha$-L-galactopiranosídeo. A modificação do ambiente químico na molécula de galactose causada por mudança de uma anomericidade $\beta$ para $\alpha$ promove um deslocamento químico paramagnético do sinal de C-1 (3,3 ppm) no espectro de $\mathrm{RMN}$ de ${ }^{13} \mathrm{C}$, o que permite diferenciar e sugerir a presença destes dois anômeros na amostra. Os sinais em 102,7 e 100,7 ppm podem

Tabela 2. Composição monossacarídica ( $\mathrm{mol} \%)$ e análise de sulfato $\left(\mathrm{NaSO}_{3} \%\right)$ das frações obtidas pelo fracionamento da amostra EXO em coluna DEAE-Sephacel

\begin{tabular}{ccccccccccc}
\hline Frações & Glc & Gal & Man & Xyl & Fuc & Rha & Ara & GalA & GalNAc & Sulfato \\
\hline EXO-0,1 & 12 & 64 & 4 & 3 & 2 & 6 & 8 & - & - \\
EXO-0,2 & 14 & 46 & 2 & TR & 4 & 15 & 10 & 7 & 3 \\
EXO-1,0 & 8 & 8 & 30 & TR & 41 & 10 & - & 2 & - & 1,4 \\
\hline
\end{tabular}

$\mathrm{TR}=$ traços, $-\mathbf{=}$ não detectado 
ser atribuídos a unidades de $\beta$-glicose ${ }^{23}$ e a unidades de $\alpha$-glicose,${ }^{24}$ respectivamente. Similar ao discutido para galactose, ocorre um deslocamento químico paramagnético de 2,0 ppm entre o anômero alfa e beta da glicose no espectro de RMN de ${ }^{13} \mathrm{C}$. Sinais em 61,2 ppm no espectro de $\mathrm{RMN}$ de ${ }^{13} \mathrm{C}$ são característicos de carbonos primários, como é o caso de carbonos 6 de hexoses. Somando esse dado ao espectro de RMN de ${ }^{13} \mathrm{C}$ DEPT135 obtido para esta amostra, o qual promove a inversão do sinal de carbonos primários, torna-se possível a atribuição deste sinal ao carbono 6 das hexoses presentes na amostra (galactose, manose e glicose).$^{25}$

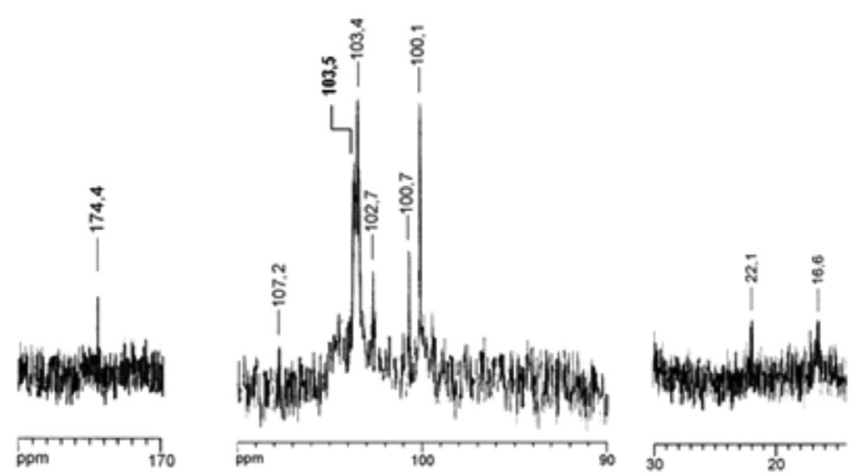

Figura 3. Espectro de $R M N$ de ${ }^{13} \mathrm{C}$ da fração EXO-0,1. Deslocamentos químicos em ppm. Calibração interna acetona $(30,200$ ppm)

A heterogeneidade e a maior intensidade dos sinais no espectro de $\mathrm{RMN}$ de ${ }^{13} \mathrm{C}$, referentes à galactose, quando comparados aos da glicose, sugerem uma mistura de dois polímeros principais, uma galactana em maior proporção e uma glucana. Esta galactana pode ser formada de unidades de $\beta$-D-Gal 3- $O$ substituída e unidades de $\alpha$-L-Gal 4- $O$ substituída, onde os sinais atribuídos ao C-1 são 103,5 e 100,2 ppm, respectivamente. ${ }^{26} \mathrm{O}$ sinal atribuído ao C-1 de $\beta$-D-Gal sofre um deslocamento químico paramagnético no espectro de RMN de ${ }^{13} \mathrm{C}$ para $104,8 \mathrm{ppm}$, quando ligada a unidades de $\alpha$-D-Galactose $(96,1 \mathrm{ppm}){ }^{26}$

A presença do grupamento metil $\left(-\mathrm{CH}_{3}\right)$ de unidades de ramnose é observada em 16,6 ppm. ${ }^{22}$ A ausência de ácido urônico na fração EXO-0,1 e a presença de sinais em 174,4 e 22,1 ppm sugerem a presença de ácido pirúvico e/ou acetil. Os sinais em 23,7 e 175,2 ppm, referentes ao grupo metil e ao carbono carbonílico de acetal de ácido pirúvico, respectivamente, são atribuídos a unidades de $\beta$-galactose piruvatadas em C-3 e C- $4 .{ }^{27} \mathrm{O}$ sinal em 107,2 ppm sugere substituição por acetal de ácido pirúvico, esterificando os carbonos 3 e 4 de unidades de $\beta$-galactose (material complementar). Segundo Ravenscroft, ${ }^{27}$ quando o ácido pirúvico está ligado ao C-3 e C-4, o anel formado nessa estrutura assemelha-se a um anel furanosídico, deslocando o sinal referente ao C-2 do acetal pirúvico no espectro (107,23 ppm). Todavia, segundo Gorin e colaboradores, ${ }^{28}$ o tempo de relaxação do carbono acetálico pode impedir sua detecção por espectroscopia de $\mathrm{RMN}$ de ${ }^{13} \mathrm{C}$, mesmo quando este substituinte está presente em uma quantidade significativa, o que, então, não descarta a possibilidade do sinal ser também resultante da presença de unidades furanosídicas de arabinose, uma vez que este monossacarídeo está presente na amostra. ${ }^{29}$

Este é o primeiro relato na literatura da presença de ácido pirúvico em polissacarídeos de diatomáceas. O ácido pirúvico ligado como acetal cíclico a uma unidade glicosil é comum em polissacarídeos extracelulares de bactérias; frequentemente se encontra ligado a C-4 e C-6 de unidades de D-glicopiranose, D-manopiranose ou D-galactopiranose. Estas unidades podem ser terminais ou estarem no meio da cadeia. ${ }^{30}$

\section{Análise química parcial da fração EXO-0,2}

A fração EXO-0,2 é constituída principalmente de galactose, ramnose, glicose e arabinose. Possui, ainda, na sua composição ácido galacturônico, $\mathrm{N}$-acetil-galactosamina e pequena porcentagem de sulfato. A composição monossacarídica e o espectro de RNM de ${ }^{13} \mathrm{C}$ dessa amostra mostram um perfil diferente da fração anterior (Figura 4).
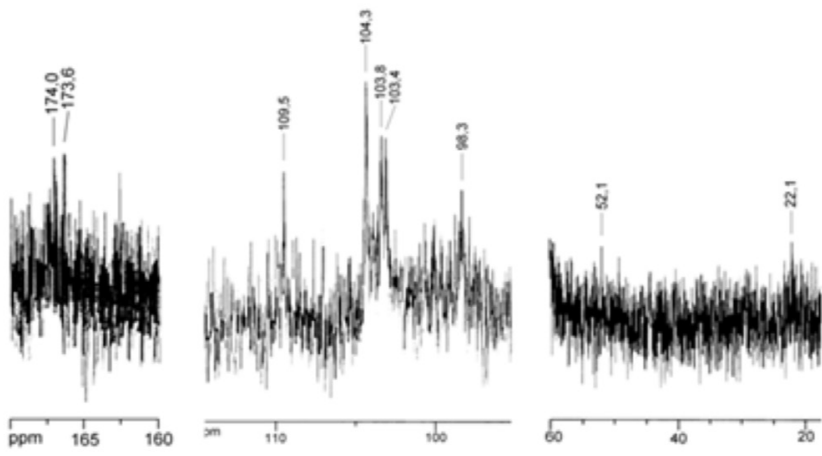

Figura 4. Espectro de RMN de ${ }^{13} \mathrm{C}$ da fração EXO-0,2. Deslocamentos químicos em ppm. Calibração interna acetona (30,200 ppm)

Na região anomérica do espectro de $\mathrm{RMN}$ de ${ }^{13} \mathrm{C}$ desta fração, os sinais em 104,3 e 103,8 ppm são atribuídos a unidades de $\beta$-D-galactopiranose, levando-se em consideração maior porcentagem de galactose na amostra. ${ }^{22}$ Deslocamentos químicos paramagnéticos referentes à arabinose indicam sua forma furanosídica e configuração $\alpha$. O sinal em 109,5 ppm presente no espectro de RMN de ${ }^{13} \mathrm{C}$ pode ser atribuído ao $\mathrm{C}-1$ de unidades terminais não redutores de $\alpha$-arabinofuranose. ${ }^{29}$

Observa-se ainda a presença do sinal em 16,6 ppm, característico de grupamento metil da ramnose. ${ }^{22} \mathrm{O}$ sinal em $98,3 \mathrm{ppm}$ indica $\mathrm{C}-1$ das unidades de $\alpha$-ácidos urônicos. ${ }^{31}$ Os sinais em 174,0 a 173,6 ppm são característicos de carbonila, sendo atribuídos ao C-6 de ácido galacturônico ${ }^{22}$ e ao carbono amídico da $\mathrm{N}$-acetil-galactosamina, respectivamente. ${ }^{32}$ Este espectro mostra ainda sinais em 22,1 e 52,1 ppm, que podem ser atribuídos a metil ligado ao carbono carbonílico e do C-2 ligado a nitrogênio de $\mathrm{N}$-acetil-galactosamina, respectivamente. ${ }^{32}$

As análises dos derivados metilados da fração EXO-0,2 apresentaram grande porcentagem de terminais não redutores. Os derivados majoritários presentes nesta fração foram 2,3-Me2-Rha e 3,4-Me2-Rha, que demonstram ligações $(1 \rightarrow 4)$ e $(1 \rightarrow 2)$ nas unidades de ramnose e 2,3,6-Me3-Gal, que demonstra ligação $(1 \rightarrow 4)$ nas unidades de galactose. A presença de terminais não redutores de unidades de arabinofuranose foi demonstrada através do derivado metilado 2,3,5-Me3-Araf.

\section{Análise química parcial da fração EXO-1,0}

A análise dessa fração mostra um polímero sulfatado constituído principalmente por fucose e manose. São observadas mudanças dos sinais no espectro de $\mathrm{RMN}$ de ${ }^{13} \mathrm{C}$ dessa fração em relação às demais frações (Figura 5).

O espectro de RMN de ${ }^{13} \mathrm{C}$ da fração EXO-1,0 mostra um elevado grau de complexidade na região anomérica. Devido à grande variação de monossacarídeos e da relação 1:1 entre fucose e manose, um único sinal nessa região pode ser atribuído a mais de um tipo de unidade. Como exemplo, o sinal em 102,4 ppm que pode ser atribuído ao C-1 de $\alpha$-L-fucopiranose ${ }^{33}$ ou ao $\mathrm{C}$ - 1 de unidades terminais não redutoras de manopiranose..$^{23}$ 


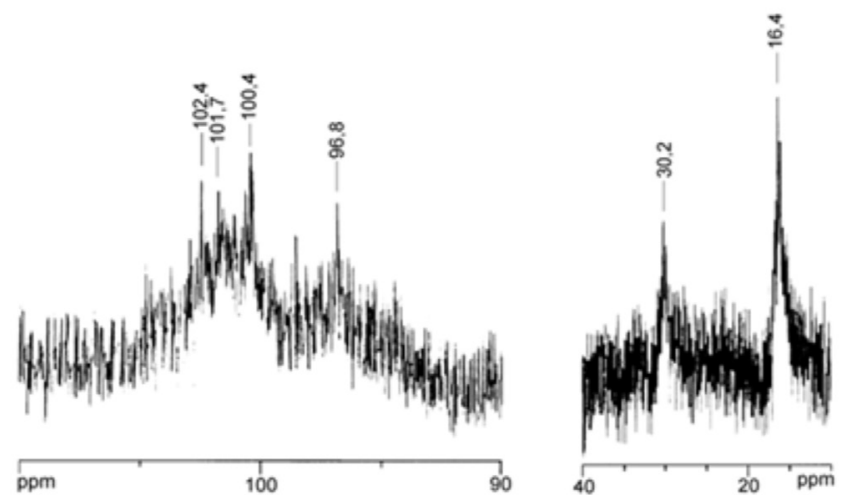

Figura 5. Espectro de RMN de ${ }^{13} \mathrm{C}$ da fração EXO-1,0. Deslocamentos químicos em ppm. Calibração interna acetona (30,200 ppm)

Outra dificuldade para atribuição de sinais é o fato dessa amostra ser sulfatada. Substituições por grupamentos sulfato causam deslocamentos químicos paramagnéticos nos sinais de $\mathrm{RMN}$ de ${ }^{13} \mathrm{C}$. Sendo assim, a heterogeneidade da fração e a provável complexidade desses polímeros dificultam uma análise química mais detalhada. Entretanto, alguns sinais característicos podem ser discutidos: a presença de grupos $O$-metil de fucose e ramnose é demonstrada em 16,2 a 16,4 ppm, respectivamente. ${ }^{22}$ Devido à baixa porcentagem de ácido urônico na amostra, não foi evidenciado o sinal referente ao C-6 do grupamento carboxílico em 174,0 ppm. Diferentemente das frações EXO-0,1 e EXO-0,2, não aparecem no espectro de RMN de ${ }^{13} \mathrm{C}$ da fração EXO-1,0 sinais em 22,1 ppm referentes a metil ligado a carbono carboxílico de ácido pirúvico e/ou N-acetil-galactosamina.

Exopolímeros contendo fucose destacam-se por sua alta viscosidade. Além disso, os desoxi açúcares apresentam propriedades hidrofóbicas, devido à presença de metil no C-6, que podem promover a formação de agregados de exopolímeros durante as florações marinhas..$^{34}$

As análises dos derivados metilados das 3 subfrações mostraram-se coerentes com os sinais atribuídos nos espectros de RMN de ${ }^{13} \mathrm{C}$. Porém, a não homogeneidade observada por cromatografia de exclusão estérea não permite propor estrutura fina dos polímeros analisados, o que poderia ser contestado. Entretanto, uma vez que foram determinadas a condição de cultivo e a extração dos polissacarídeos da microalga, a elucidação das estruturas finas desses polissacarídeos é possível de ser realizada em futuros estudos.

\section{CONCLUSÃO}

O isolamento e o cultivo da diatomácea $C$. wailesii foram realizados sob condições controladas, possibilitando estabelecer sua curva de crescimento. Durante a fase exponencial de desenvolvimento da microalga foram obtidas duas frações de polissacarídeos, exopolissacarídeos e polissacarídeos da cápsula mucilaginosa, com composição monossacarídica, teores de açúcar total, ácido urônico e grupamentos sulfatos distintos. A composição lipídica da microalga C. wailesii apresentou somente ácidos graxos saturados. $\mathrm{O}$ fracionamento da amostra de exopolissacarídeo forneceu três subfrações. Esses resultados são inéditos para o gênero Coscinodiscus, sendo sugeridas as estruturas químicas parciais através de técnica de RNM de ${ }^{13} \mathrm{C}$ e metilação. A fração EXO-0,1 demonstrou ser constituída principalmente por uma galactana com esterificações por ácido pirúvico. A fração EXO-0,2 demonstrou ser composta principalmente por uma galactana, além de apresentar unidades de ácido galacturônico e $\mathrm{N}$-acetil-galactosamina, sendo a primeira vez que se relata a presença de açúcar aminado em polissacarídeos de diatomáceas. A fração
EXO-1,0 demonstrou ser composta principalmente por um polissacarídeo sulfatado constituído de fucose e manose, ou dois polímeros distintos, sendo uma fucana e uma manana. Esses resultados são de suma importância, uma vez que, com exceção do polissacarídeo de reserva (crisolaminarana), pouco se conhece sobre a estrutura química de polissacarídeos de diatomáceas.

\section{MATERIAL SUPLEMENTAR}

Fotomicroscopia da microalga C. wailesii cultivada evidenciando a natureza ácida de seus exopolissacarídeos, através de coloração de Alcian Blue, bem como o perfil cromatográfico obtido por CLAE da curva de hidrólise dos exopolissacarídeos de $C$. wailesii no tempo de $18 \mathrm{~h}$ e figura ilustrativa da atribuição de sinais de $\mathrm{RMN}$ de ${ }^{13} \mathrm{C}$ para acetal de ácido pirúvico esterificando C-3 e C-4 de unidades de $\beta$-galactose estão disponíveis em http://quimicanova.sbq.org.br, na forma de arquivo PDF, com acesso livre.

\section{AGRADECIMENTOS}

Ao Conselho Nacional de Desenvolvimento Científico e Tecnológico (CNPq) e ao PRONEX-Carboidratos pelos apoios financeiros concedidos. Ao Prof. Dr. L. F. Fernandes do Departamento de Botânica, UFPR, por ter cedido gentilmente a microalga.

\section{REFERÊNCIAS}

1. Fukao T.; Kimoto K.; Yamatogi T.; Yamamoto K.; Yoshida Y.; Kotani Y.; Fish. Sci. 2009, 75, 1007; Radic, T.; Kraus R.; Fuks D.; Radic, J.; Pecar, O.; Sci Total Environ. 2005, 353, 151.

2. Nagai, S.; Hori, Y.; Manabe, T.; Imai, I.; Phycologia 1995, 34, 533.

3. Rick, H. J.; Dürselen, C. D.; Helgolaender Meeresuntersuchunge 1995, 49, 355.

4. Fernandes, L. F.; Zehnder-Alves, L.; Bassfeld, J. C.; Phycol. Res. 2001, $49,89$.

5. Myklestad, S.; Haug, A.; J. Exp. Marine Biol. Ecol. 1972, 9, 125; Staats, N.; Stal, L. J.; Mur, L. R.; J. Exp. Marine Biol. Ecol. 2000, 249, 13.

6. Brouwer, J. F. C.; Stal, L. J.; J. Phycol. 2002, 38, 464.

7. Boalch, G. T.; Hydrobiologia 1984, 116/117, 449.

8. Guillard, R. R. L. Em Culture of Marine Invertebrate Animals; Smith, W. L.; Chanley, M. H., eds.; Plenum Press: New York, 1975, p. 26; Guillard, R. R. L; Ryther, J. H.; Can. J. Microbiol. 1962, 8, 229.

9. Nagai, S.; Imai, I.; Manabe, T.; J. Plankton Res. 1998, 20, 1417.

10. Shapiro, L. Em Microbiology: dynamics e diversity; Perry, J. J.; Stanley, J. T., eds.; Saunders College Publising: New York, 1997.

11. U. S. EPA; National Eutrophication Reserch Program, 1971, Northwest Water Laboratoy: Corvallis.

12. Bradford, M. M.; Anal. Biochem. 1976, 72, 248.

13. Dubois, M.; Gilles, K. A.; Hamilton, J. K.; Rebers, P. A.; Smith, F.; Anal. Chem. 1956, 28, 350.

14. Dodgson, K. S.; Biochem. J. 1961, 78, 312.

15. Filizetti-Cozz, T. M. C. C.; Carpita, N. C.; Anal. Biochem. 1991, 197, 157.

16. Ciucanu, I.; Kerek, F.; Carbohydr. Res. 1984, 131, 209.

17. Saeman, J. F.; Harris, E. E.; Kline, A. A.; Ind. Eng. Chem. 1954, 17, 95.

18. Bhosle, N. B.; Sawant, S. S.; Garg, A.; Wagh, A. B.; Evans, L. V.; Biofouling 1996, 10, 301; Bhosle, N. B.; Sawant, S. S.; Garg, A.; Wagh, A. B.; Bot. Mar. 1995, 38, 103.

19. Alldredge, A. L.; Passow, U.; Logan, B.; Deep Sea Res. 1993, 40, 1131.

20. Daniel, G. F.; Chamberlain, J. E. B. G.; British Phycol. J. 1987, $22,101$.

21. Percival, E.; Rahmasn, M. A.; Weigel, H.; Phytochemistry 1980, 19, 809.

22. Gorin, P. A. J.; Mazurek, M.; Can. J. Chem. 1975, 53, 1212. 
23. Alquini, G.; Carbonero, E. R.; Rosado, F. R.; Cosentino, C.; Iacomini, M.; FEMS Microbiol. Lett. 2003, 18, 1.

24. Gorin, P. A. J.; Baron, M.; Iacomini, M.; CRC Handbook lichenology, Florida, 1988, vol. 3, p. 9.

25. Prado, S. R. T.; Gorin, P. A. J.; Stuelp, P. M.; Honda, N. K.; Iacomini, M.; Carbohyd. Polym. 1999, 40, 271.

26. Miller, I. J.; Furneaux, R. H.; Bot. Mar.1996, 39, 141.

27. Chiovitti, A.; Bacic, A.; Craik, D. J.; Munro, S. L. A.; Kraft, G. T.; Liao, M. L.; Carbohydr. Res. 1997, 299, 229; Ravenscroft, N.; Lesley-Parolis, A. S.; Parolis, H.; Carbohydr. Res. 1994, 254, 333.

28. Gorin, P. A. J.; Mazurek, M.; Duarte, H. S.; Iacomini, M.; Duarte, J. H.; Carbohydr. Res. 1982, 100, 1.

29. Dong, Q.; Fang, J.; Carbohydr. Res. 2001, 332, 109.
30. Garegg, P. J.; Jansson, P. E.; Lindberg, B.; Lindh, F.; Lonngren, J.; Carbohydr. Res. 1980, 78, 127.

31. Renard, C. M. G. C.; Lahaye, M.; Mutter, M.; Voragen, F. G. J.; Thibault, J. F.; Carbohydr. Res. 1980, 305, 271.

32. Lesley, G.; Sharples, J. L.; Stephen, G. W.; Carbohydr. Res. 1999, 319, 204.

33. Ponce, N. M. A.; Pujol, C. A.; Damonte, M. L. F.; Stortz, C. A.; Carbohydr. Res. 2003, 338, 153.

34. Vieira, A. A. H.; Ortolano, P. I. C.; Giroldo, D.; Oliveira, M. J. D.; Bittar, T. B.; Lombardi, A. T.; Sartori, A. L.; Limnol. Oceanog. 2008, 53, 1887; Giroldo, D.; Augusto, A.; Vieira, H.; Paulsen, B. S.; J. Phycol. 2003, 39, 1109. 


\section{ISOLAMENTO E ANÁLISE QUÍMICA PARCIAL DE EXOPOLISSACARÍDEOS DA DIATOMÁCEA MARINHA CULTIVADA Coscinodiscus wailesii (COSCINODISCALES, BACILLARIOPHYTA)}

Poliana G. Marson-Ascêncio* e Sérgio Donizeti Ascêncio

Universidade Federal do Tocantins, Campus Universitário de Palmas, Av. NS15, B1. BALA-Medicina, 77020-210 Palmas - TO, Brasil Selma Faria Zawadzki Baggio

Departamento de Bioquímica e Biologia Molecular, Setor de Ciências Biológicas, Universidade Federal do Paraná, Centro Politécnico, CP 19046, 81531-990 Curitiba-PR, Brasil

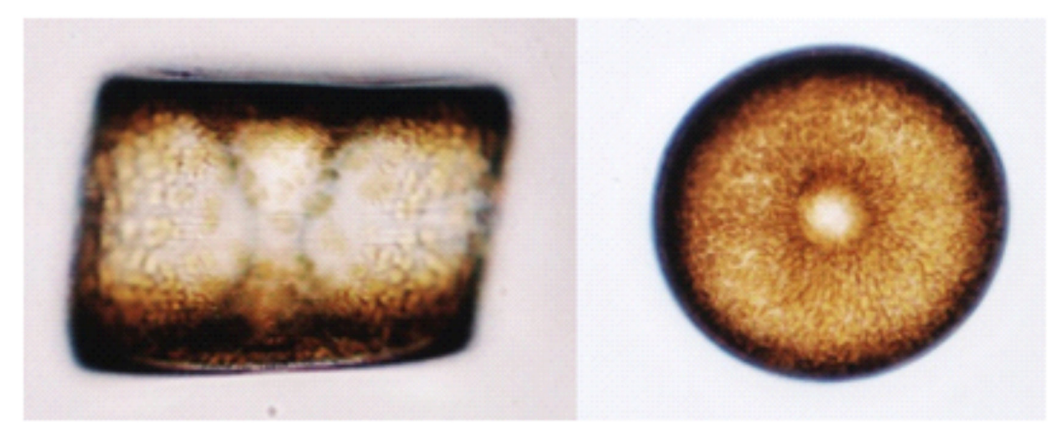

Figura 1S. Fotomicroscopia da microalga C. wailesii cultivada (vista lateral e frontal em aumento de $400 \mathrm{X}$ )
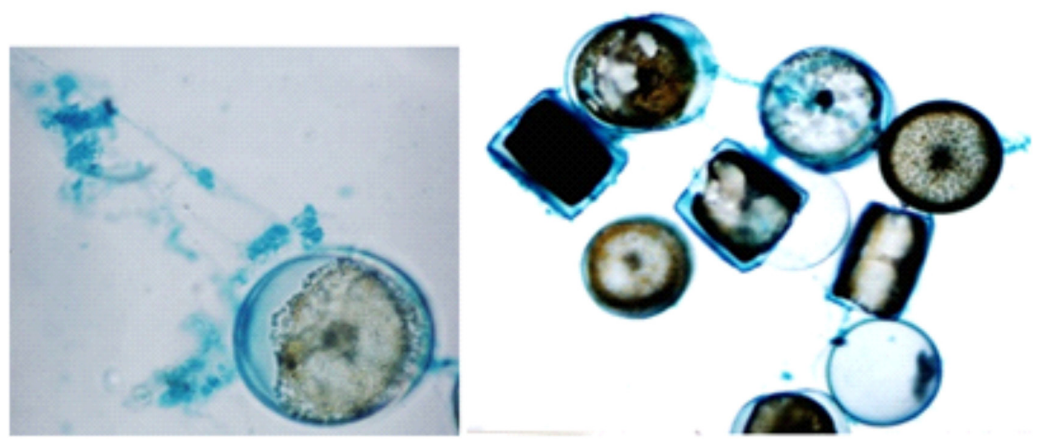

Figura 2 S. Fotomicroscopia da microalga C. wailesii, evidenciando a natureza ácida de seus exopolissacarídeos através de coloração de Alcian Blue

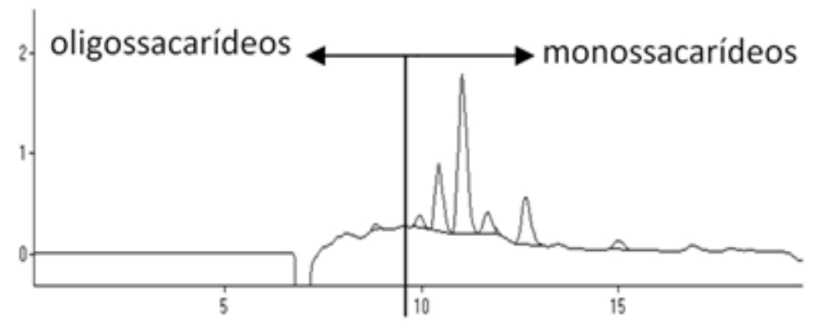

Figura 3S. Perfil cromatográfico obtido por CLAE da curva de hidrólise dos exopolissacarídeos de C. wailesii no tempo de $18 \mathrm{~h}$

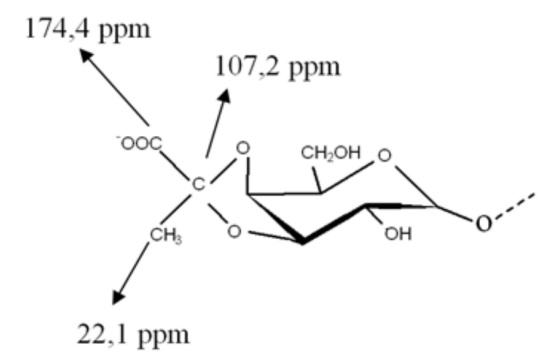

3,4-O-(1-CARBOXIETILIDENO)-GALACTOSE

Figura 4S. Atribuição de sinais de RMN de ${ }^{13} \mathrm{C}$ para acetal de ácido pirúvico esterificando $C$-3 e C-4 de unidades de $\beta$-galactose

*e-mail: polianamarson@uft.edu.br 